\title{
Mechanism of Regular Atrial Tachyarrhythmias During Combined Pulomonary Vein Isolation and Complex Fractionated Electrogram Ablation in Patients With Atrial Fibrillation
}

\author{
Gi-Byoung Nam, MD; Eun-Sun Jin, MD; HyungOh Choi, MD; Hae-Geun Song, MD; \\ Sung-Hwan Kim, MD; Ki-Hun Kim, MD; Eui-Seock Hwang, MD; \\ Kyoung-Min Park, MD*; Jun Kim, MD**; Kyoung-Suk Rhee, MD ${ }^{\dagger}$; \\ Kee-Joon Choi, MD; You-Ho Kim, MD
}

\begin{abstract}
Background: Atrial tachyarrhythmias (ATA) frequently develop during catheter ablation of atrial fibrillation (AF), but the mechanism of ATA during combined pulmonary vein isolation (PVI) and complex fractionated electrogram-guided ablation (CFEA) has not been reported.
\end{abstract}

\begin{abstract}
Methods and Results: This study involved 105 patients with symptomatic, drug-refractory AF. After PVI, CFEA was performed in the left/right atrium if $A F$ remained inducible in paroxysmal AF (PAF) or persisted in persistent AF (PeAF). For the 70 PAF patients, PVI alone rendered AF non-inducible in 29 patients (41.4\%), and converted inducible $A F$ into inducible atrial flutter $(A F I)$ in 10 patients (14.3\%). For the remaining 31 PAF patients, additional CFEA rendered AF non-inducible in 11 patients (15.7\%), whereas only AFI was inducible in 11 patients (15.7\%). For 35 PeAF patients, PVI and CFEA converted AF into sinus rhythm in 2 (5.7\%) and into AFI in 21 (60.0\%) patients, while AF persisted in 12 patients (34.3\%). The mechanism of ATA was focal (20/114, 17.5\%), roofdependent $(20 / 114,17.5 \%)$, peri-mitral $(33 / 114,28.9 \%)$, cavotricuspid isthmus-dependent $(34 / 114,29.8 \%) \mathrm{AFI}$ or unknown (7/114, 6.1\%). Successful ablation was achieved in 93/114 (81.6\%) tachycardias.
\end{abstract}

Conclusions: The major mechanism of ATA during the combined approach of PVI and CFEA is macroreentry around large anatomic obstacles such as the pulmonary vein or the mitral or tricuspid annuli. (Circ J 2010; 74: 434-441)

Key Words: Atrial fibrillation; Atrial flutter; Catheter ablation; Heart atria; Tachycardia

$\mathbf{R}$ ecent advances in catheter ablation has lead to widespread application of atrial fibrillation (AF) ablation in clinical practice. ${ }^{1-6}$ Based on data from basic and clinical research, ${ }^{4,5}$ a tailored approach targeting complex fractionated electrograms (CFE) has been proposed..$^{7-9} \mathrm{~A}$ combined approach of electrogram-guided CFE ablation (CFEA) and pulmonary vein isolation (PVI) could provide additional benefit in achieving the end-point of the procedure or long-term maintenance of sinus rhythm in catheter ablation of AF.

One of the clinical challenges during catheter ablation of $\mathrm{AF}$ is the occurrence of regular atrial tachyarrhythmias (ATA) that are often difficult to deal with. The aim of the present study was to define the mechanism of atrial tachycardias occurring during combined PVI and CFEA.

\section{Study Group}

This prospective study comprised 105 consecutive patients with symptomatic, drug-refractory ( $\geq 1$ class I, III antiarrhythmic drugs) paroxysmal AF (PAF) or persistent $\mathrm{AF}$ (PeAF). Patients' clinical and demographic features are summarized in Table 1. PAF and PeAF were defined as pre-

Received August 25, 2009; revised manuscript received November 10, 2009; accepted November 15, 2009; released online January 14, 2010 Time for primary review: 43 days

Asan Medical Center, Department of Internal Medicine, University of Ulsan College of Medicine, *Sanggye Paik Hospital, Department of Internal Medicine, College of Medicine, Inje University, Seoul, **Pusan National University Yangsan Hospital, Department of Internal Medicine, Pusan National University School of Medicine, Busan and Chonbuk National University Hospital, Division of Cardiology, Department of Internal Medicine, Chonbuk National University, Chonju, Republic of Korea

Mailing address: Gi-Byoung Nam, MD, Department of Internal Medicine, University of Ulsan College of Medicine, Poongnap-dong 388-1, Songpa-gu, Seoul 138-736, Republic of Korea. E-mail: gbnam@amc.seoul.kr

ISSN-1346-9843 doi:10.1253/circj.CJ-09-0622

All rights are reserved to the Japanese Circulation Society. For permissions, please e-mail: cj@j-circ.or.jp 


\begin{tabular}{lcc|}
\hline Table 1. Clinical Characteristics of the Study Patients & & \\
& Paroxysmal AF ( $\mathbf{n}=\mathbf{7 0})$ & Persistent AF $(\mathbf{n}=\mathbf{3 5})$ \\
Age (years) & $54 \pm 11(35-78)$ & $52 \pm 11(31-74)$ \\
Sex (M/F) & $55 / 15$ & $27 / 8$ \\
Past history of ischemic stroke & 4 & 3 \\
Hypertension & 24 & 12 \\
Hypertensive heart disease & 11 & 5 \\
Diabetes mellitus & 8 & 5 \\
Hyper/hypothyroidism & $0 / 2$ & $1 / 0$ \\
Coronary artery disease & 4 & 3 \\
Percutaneous coronary intervention or bypass graft & 3 & 3 \\
Congestive heart failure (NYHA $\geq 2)$ & 2 & 6 \\
Ejection fraction (\%) & 29.6 (range 46-70) \\
Left atrial dimension (mm) & $53.7 \pm 8.3$ (range 29-68) \\
Ejection fraction $<50 \%$ & $40.5 \pm 5.3($ range 29-54) & $44.2 \pm 5.2$ (range 36-55) \\
Follow-up & 2 & 9 \\
\hline
\end{tabular}

AF, atrial fibrillation; NYHA, New York Heart Association functional class.

viously described. ${ }^{10}$ Anticoagulation was maintained using an international normalized ratio $>1.8$ at least 4 weeks before the procedure. Transesophageal echocardiography was performed on the day of or the day prior to the procedure. The study protocol was reviewed and approved by the Institutional Committee on Human Research at Asan Medical Center and each patient provided written informed consent.

\section{Electrophysiology Study}

After discontinuation of all antiarrhythmic agents for at least 5 half-lives, an electrophysiological study was performed in the fasting state, typically under sedation with a continuous infusion of propofol $(200-600 \mathrm{mg} / \mathrm{h})$. Patients taking amiodarone were instructed to discontinue medication 1 month prior to the procedure. A $6 \mathrm{~F}$ quadripolar catheter and a $7 \mathrm{~F}$ decapolar catheter were introduced percutaneously through the femoral and jugular veins and placed in the His-bundle and coronary sinus (CS) regions, respectively. Two 8F sheaths (SL1, St Jude Medical, Inc) were introduced into the left atrium (LA) using a modified Brockenbrough technique. After transseptal puncture, intravenous heparin was injected as a bolus $(80 \mathrm{IU} / \mathrm{kg})$ and intermittently $(1,000-2,000 \mathrm{IU} / \mathrm{h})$ to maintain an activated clotting time $>250$ s. In addition, heparinized saline was continuously infused through the transseptal sheath $(3 \mathrm{ml} / \mathrm{min})$ to avoid formation of thrombi or air emboli. The infusion rate was increased to $17 \mathrm{ml} / \mathrm{min}(\leq 30 \mathrm{~W})$ or $30 \mathrm{ml} / \mathrm{min}(>30 \mathrm{~W})$ during radiofrequency $(\mathrm{RF})$ application.

Multiple ECG leads (leads I, $\mathrm{a} \mathrm{V}_{\mathrm{F}}$ and $\mathrm{V}_{1}$, filtered between 0.05 and $100 \mathrm{~Hz}$ ) and intracardiac bipolar electrograms (filtered between 30 and $500 \mathrm{~Hz}$ ) were simultaneously displayed and recorded on a digital electrophysiologic recording system (CardioLab ${ }^{\circledR}$, Prucka Eng., Houston, TX, USA). The stimuli were delivered using a programmable digital stimulator (DTU-215, Bloom \& Associates, Reading, PA, USA) at twice the diastolic threshold and with a 2-ms pulse width for conventional pacing, and at 10-20 mA for high output pacing. Conventional fluoroscopic mapping was used in 65 patients, and 3-dimensional (3D) mapping in 40 patients (NavX, ver 6.0 or 7.0, St Jude Medical, $n=36$ or Carto system, Biosense-Webster, $\mathrm{n}=4$ ).

\section{Circumferential PVI}

PVI was performed $5-10 \mathrm{~mm}$ outside the PV ostia, with isolation of 2 ipsilateral veins in 1 circumferential lesion. Ablation in the LA was performed using an irrigated catheter with a target temperature of $50^{\circ} \mathrm{C}$, maximal power of $30 \mathrm{~W}$, and an infusion rate of $17 \mathrm{ml} / \mathrm{min}$. Power was temporarily increased to $40 \mathrm{~W}$ in an anterior circumference resistant to RF application. The endpoint of PVI was elimination or dissociation of the PV potentials (PVP). An ostial or carinal touch-up was additionally performed if PVP remained after PVI without an obvious gap along the line. Ablation at the PV ostia and within the CS was performed at $15-25 \mathrm{~W}$ and $50^{\circ} \mathrm{C}$. Energy was applied for $40-60 \mathrm{~s}$ at each point until the loss of antral potential or the sharp component of the bipolar electrogram.

\section{Study Protocol and CFEA}

In patients with $\mathrm{PAF}, \mathrm{AF}$ was induced before the procedure using a 5-s burst pacing from the distal and proximal CS and the right atrial electrodes. Pacing was begun at a cycle length (CL) of $250 \mathrm{~ms}$ and the CL was reduced in 10-ms intervals until atrial refractoriness or a CL of $200 \mathrm{~ms}$ was reached. Inducibility was defined as induction of AF lasting $>2 \mathrm{~min}$.

After completion of PVI, LA ablation targeting CFE was commenced by searching for the CFE from the posterior wall, the roof and the septum followed by the mitral annulus and base of the LA appendage (LAA). CFE was defined visually as a highly fractionated, nearly continuous electrogram. AF was re-induced if it terminated during the procedure, and abnormal electrograms were examined again if the re-induced $\mathrm{AF}$ lasted $>2 \mathrm{~min}$. If $\mathrm{AF}$ persisted or remained inducible after LA lesions, RF application was continued inside the CS and in the right atrium (RA), especially at the cavotricuspid isthmus, superior vena cava, crista terminalis, and the RA septum. The procedure endpoint was non-inducibility.

In patients with PeAF, CFEA was continued after PVI until AF termination, and the procedure endpoint was termination to sinus rhythm. In the last 3 patients with PeAF, a NavX-automated CFE detection algorithm was used. Automated CFE maps were created based on CFE CLs in an 8-s window, P-P sensitivity of $0.1 \mathrm{mV}$, width slider of $24 \mathrm{~ms}$ and refractory period of $49 \mathrm{~ms}$.

\section{Management of Regular Tachycardias}

If a regular atrial tachycardia was induced or converted from AF during CFEA, activation mapping and multiple entrain- 

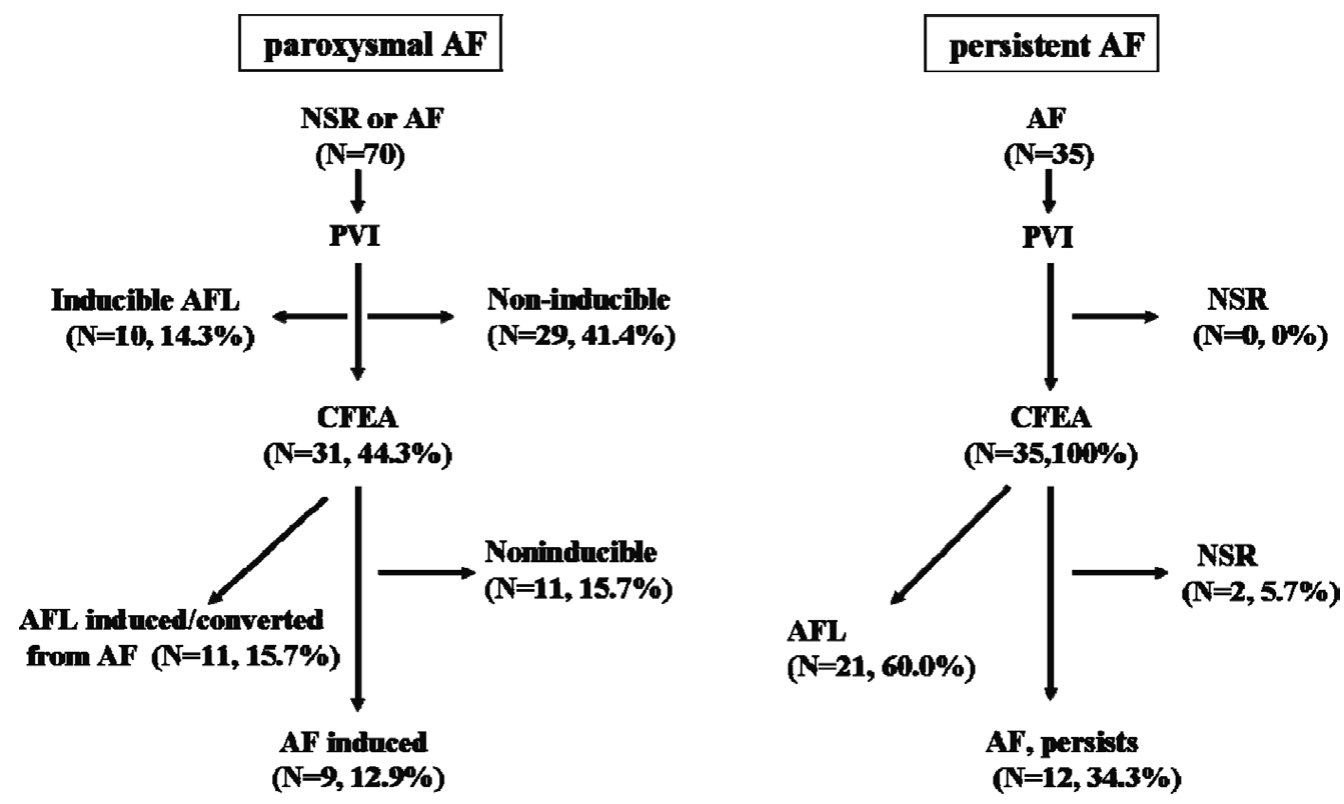

Figure 1. Results of combined PVI and CFEA in patients with paroxysmal and persistent atrial fibrillation. AF, atrial fibrillation; $\mathrm{AFI}$, atrial flutter; CFEA, continuous fractionated electrogram-guided ablation; PVI, pulmonary vein isolation.

\begin{tabular}{lccc|} 
Table 2. Distribution of Fractionated Atrial Electrograms & $\begin{array}{c}\text { Targeted for Ablation and Sites of AF Termination } \\
\text { Paroxysmal AF } \\
(\mathbf{n = 3 1 )}\end{array}$ & $\begin{array}{c}\text { Persistent AF } \\
(\mathbf{n}=\mathbf{3 5})\end{array}$ & $\begin{array}{c}\text { Termination of AF into } \\
\text { sinus rhythm or flutter } \\
\text { (in PeAF) }\end{array}$ \\
Roof & 13 & 25 & 2 \\
Septum, left & 13 & 25 & 3 \\
Mitral annulus & 17 & 24 & 2 \\
LAA base & 11 & 22 & 7 \\
Posterior wall & 1 & 9 & 3 \\
Anterior wall & 4 & 10 & 1 \\
CS os & 8 & 8 & 1 \\
CS inside & 7 & 18 & 3 \\
Crista terminalis & 7 & 7 & 0 \\
Septum, right & 4 & 7 & 1 \\
RAA base & 2 & 1 & 0 \\
SVC isolation & 7 & 3 & 0 \\
Tricuspid annulus & 2 & 0 & 0 \\
Total & 113 & 159 & 23 \\
Mean \pm SD & $3.4 \pm 2.2$ & $4.5 \pm 1.8$ & \\
\hline
\end{tabular}

AF, atrial fibrillation; PeAF, persistent AF; LAA, left atrial appendage; CS, coronary sinus; RAA, right atrial appendage; SVC, superior vena cava.

ment mapping were performed to identify its mechanism and location. Focal or isthmus ablation was performed accordingly to eliminate the tachycardia. Atrial tachycardias that could not be easily mapped and localized or were resistant to RF applications were cardioverted. Also, AF persistent after PVI and CFEA was electrically cardioverted at the end of the procedure.

Reentrant peri-mitral atrial flutter (AFl) was defined as regular atrial tachycardia with matching PPI (PPI-TCL $<20 \mathrm{~ms}$ ) in at least 2 peri-mitral regions. Roof-dependent $\mathrm{AFl}$ was diagnosed when PPI matched TCL in the posterior wall and in the roof or anterior wall. When the entrainment mapping results were not compatible with the above reentrant $\mathrm{AFl}$, and the activation sequence showed a centrifugal propagation from a limited area of atrium, focal ablation targeting the earliest activation was performed. A focal mechanism in our study was confirmed using the following criteria: (1) failure to meet the entrainment result in $>2$ atrial regions, (2) centrifugal propagation of activation, and (3) termination by focal ablation. However, the term "focal" does not differentiate between true focal automatic tachycardia and focal micro-reentry. 
A II

III

aVF

v1

ABL p

ABL d

HIS p

HIS d

CS 9,10

CS 7,8

CS 5,6

$\operatorname{CS} 3,4$

CS 1,2

B II

III

aVF

V1

ABL $p$

ABL d

CS 9,10

$\operatorname{CS} 7,8$

CS 5,6

CS 3,4

CS 1,2

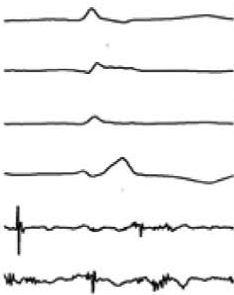

r r
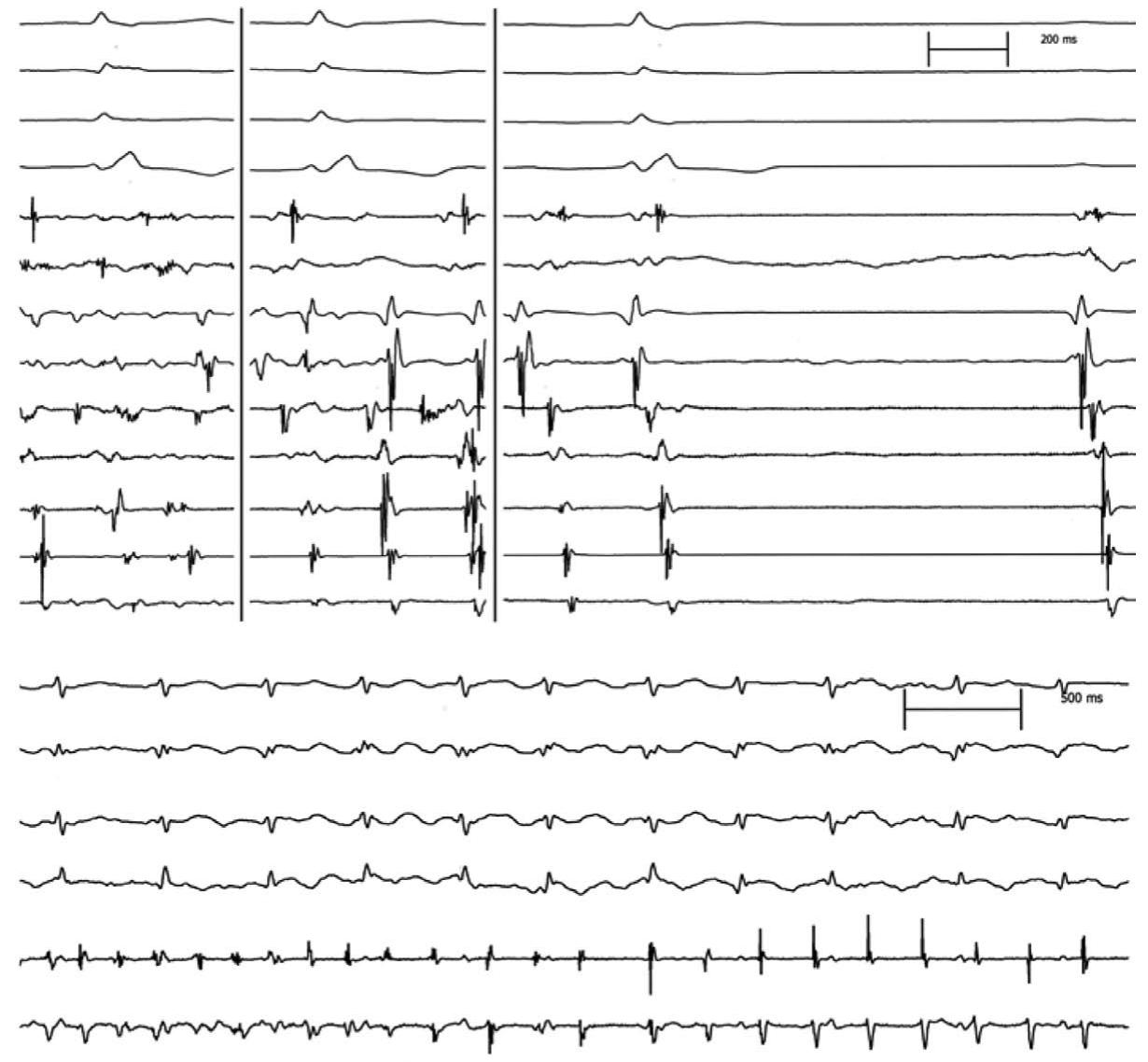

(1)
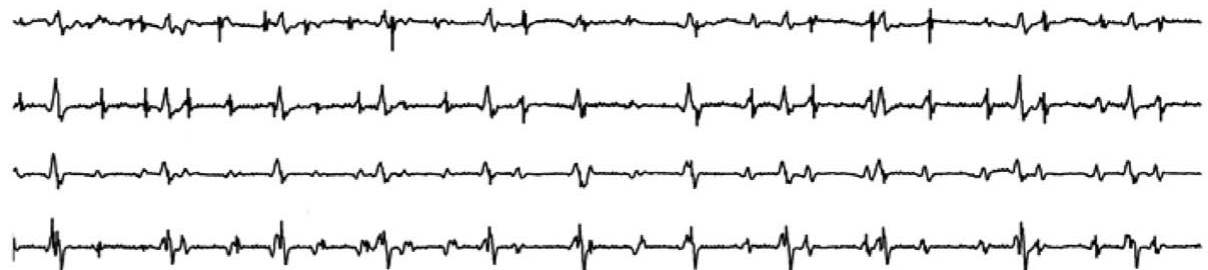

Figure 2. Effect of CFEA in patients with PeAF. Surface ECGs from leads $I$, III, aV $\mathrm{F}_{\mathrm{F}}$, and $\mathrm{V}_{1}$ and intracardiac electrograms from the coronary sinus, Lasso and ABL catheters are shown. (A) A 31-year-old male patient with PeAF. Post-PVI CFE remain in the roof, mitral annulus, septum, LAA base, and inside the coronary sinus. During RF application in the septum, AF terminated directly into sinus rhythm after prolongation of the AF CL in the ABL catheter electrograms. (B) A 38 year-old male patient with PeAF. Post-PVI CFE remained in the septum, roof and inside the coronary sinus. AF was converted into AFI during RF application inside the coronary sinus region. ABL, ablation; AF, atrial fibrillation; AFI, atrial flutter; CFE, complex fractionated electrograms; CFEA, continuous fractionated electrogram-guided ablation; CL, cycle length; LAA, left atrial appendage base; PeAF, persistent AF; PVI, pulmonary vein isolation; RF, radiofrequency.

\section{Post-Ablation Care and Follow-up}

Subcutaneous low-molecular-weight heparin and warfarin were administered for 3 days commencing on the evening of the procedure day. Warfarin was continued for $>3$ months and ceased if the patient did not have risk factors for thromboembolism. Anti-arrhythmic drugs were continued for 1-3 months in patients with a history of PeAF and were re-initiated if symptomatic AF recurred in patients with PAF.

Patients were seen in the outpatient clinic at 1-month intervals for the first 3 months, and then every 2-3 months thereafter. ECGs were checked on each visit and, if indicated, a
Holter or event recording was used for detection of recurrent AF.

All patients were advised to visit outpatient clinics or emergency rooms for symptomatic episodes of palpitations, and in cases of persistent arrhythmia episodes, to obtain ECG documentation of the underlying rhythm. The absence of $\mathrm{AF}$ and $\mathrm{AFl}$ was confirmed in all asymptomatic patients with serial ECGs and/or 24-h Holter recordings. A successful outcome over the follow-up period was defined as the lack of electrocardiographically recorded AF, and no PAF on Holter or event recording, and subjective symptomatic improvement 


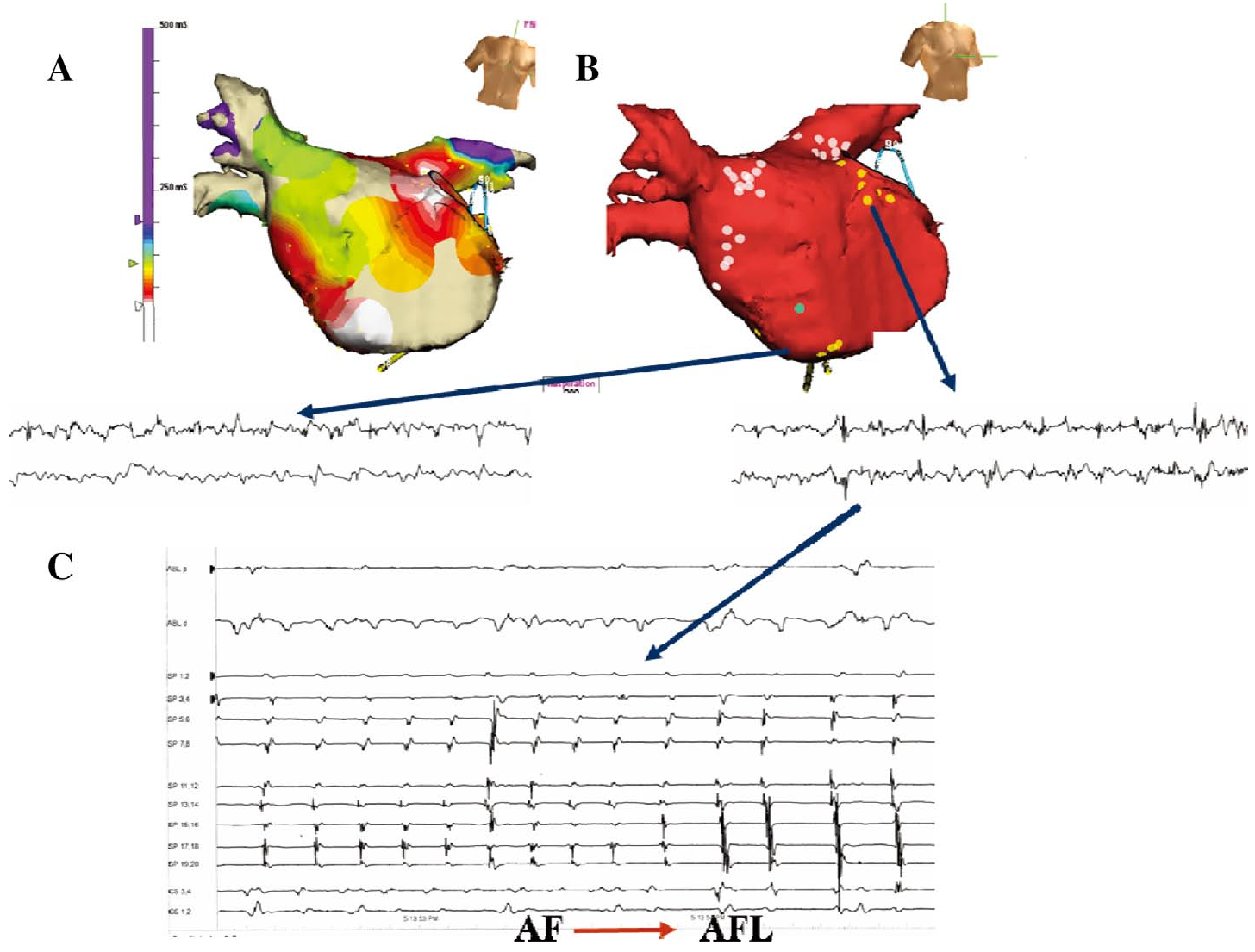

Figure 3. Localization of CFE using 3D automated CFE detection program. In the last 3 patients with PeAF, an automated 3D CFE detection program was used for localization of CFE. (A) CFE map obtained from a 52-year-old female patient with PeAF. Post-PVI CFE was localized in the low septal region and near the LAA base (white color). (B, C) During RF application in the LAA base followed by RF in the low septum, AF was converted into AFI. AFI was again eliminated by linear lesion in the roof and in the LA isthmus. AF, atrial fibrillation; AFI, atrial flutter; CFE, complex fractionated electrogram; 3D, 3-dimensional; LAA, left atrial appendage; PeAF, persistent AF; PVI, pulmonary vein isolation; RF, radiofrequency.

after an 3-month blanking period. ${ }^{11,12}$

\section{Data Analysis}

Data are expressed as mean \pm SD. Student's t-test was used for statistical comparison. A P-value $<0.05$ was considered to indicate significant difference.

\section{Results of RF Ablation}

For the 70 PAF patients, PVI alone rendered AF non-inducible in $29(41.4 \%)$, and only AFl were inducible in $10(14.3 \%)$ patients. For the remaining $31(44.3 \%)$ PAF patients, additional CFEA rendered inducible $\mathrm{AF}$ non-inducible in 11 $(15.7 \%)$ patients, and converted it into $\mathrm{AFl}$ in $11(15.7 \%)$ patients. AF persisted in $9(12.9 \%)$ patients after the combined ablation procedure.

For the $35 \mathrm{PeAF}$ patients, PVI alone failed to terminate $\mathrm{AF}$ into sinus rhythm in any patient. Additional CFEA resulted in AF being terminated into sinus rhythm in 2 of 35 $(5.7 \%)$ patients, and into AFl in 21 of $35(60.0 \%)$ patients. The additional CFEA had no effect in 12 (34.3\%) PeAF patients (Figure 1).

The duration of RF application for PVI were 3,855 $\pm 1,515 \mathrm{~s}$ for PAF and 3,978 $\pm 1,807$ for PeAF. Although the mean duration of RF application during CFEA appeared to be longer in patients with PeAF $(1,032 \pm 590$ vs $1,549 \pm 753 \mathrm{~s})$, this difference did not quite reach statistical significance $(\mathrm{P}=0.06)$.

\section{Distribution of Fractionated Electrograms and Results of CFEA}

Fractionated electrograms were more commonly associated with specific LA regions such as the roof, septum, lateral mitral annulus, and base of the LAA. Table 2 summarizes the distribution of the CFE areas targeted for CFEA. The mean number of atrial regions targeted for CFEA was higher in patients with PeAF than in patients with PAF ( $3 \pm 2$ vs $5 \pm 2$ regions, $\mathrm{P}<0.05)$. Most of the $\mathrm{RF}$ lesions targeting $\mathrm{CFE}$ were delivered in a circumscribed area, creating a coin lesion. If CFE electrograms were distributed in a large area, especially in the posterior wall, roof or septum, a linear lesion was made across the CFE area.

Post-PVI CFEA rendered inducible AF either into inducible AFl or non-inducible in patients with PAF. PeAF terminated directly to sinus rhythm in 2 patients and converted into $\mathrm{AFl}$ in 21 patients (Figures 2,3). Conversion occurred during CFEA at the LAA, CS inside, roof, septum or mitral annulus etc (Table 2). 
Table 3. Mechanisms of Atrial Tachycardia During or After PVI and CFEA

Mechanism

Focal

Peri-mitral

Roof-dependent

Right isthmus

Unknown

Total (total number/successful ablation)

11 (11)

16 (11)

$11(10)$

20 (20)

$2(0)$

60 (52)
Persistent AF
(total number/successful ablation)

9 (8)

17 (14)

9 (7)

14 (12)

$5(0)$

54 (41)
Success rate

(total number/successful ablation, \%)

$20(19,95.0 \%)$

$33(25,75.8 \%)$

$20(17,85.0 \%)$

$34(32,94.1 \%)$

$7(0,0 \%)$

$114(93,81.6 \%)$

PVI, circumferential pulmonary vein isolation; CFEA, continuous fractionated electrogram-guided ablation; AF, atrial fibrillation.

A

B

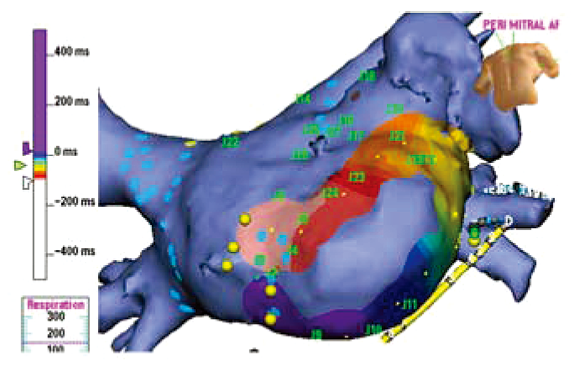

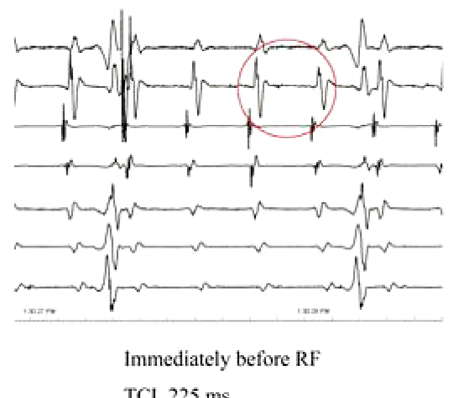

TCL $225 \mathrm{~ms}$

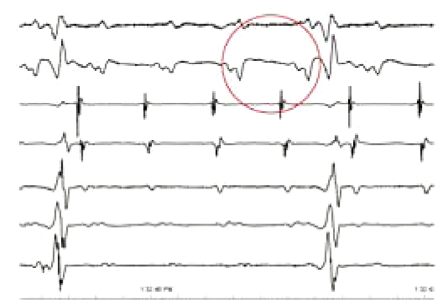

140 seconds after onset of RF

TCL 235 to $245 \mathrm{~ms}$

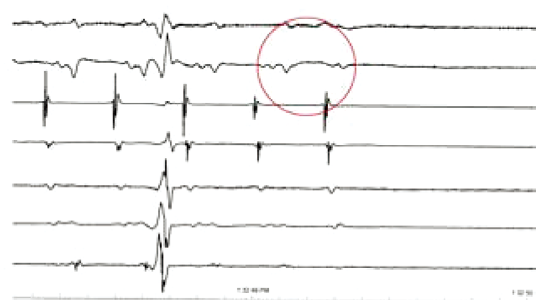

150 seconds

TCL 245 to $255 \mathrm{~ms}$ and then termination

Figure 4. Activation map of a peri-mitral flutter converted during CFEA and local electrogram change during catheter ablation. (A) A 41-year-old male patient with persistent AF. Post-PVI CFE remain in the roof, septum, anterior wall, and inside the coronary sinus. During RF application inside the CS, AF was converted into a regular atrial tachyarrhythmia. Activation sequence map suggested peri-mitral AFI as the mechanism of tachycardia. (B) During RF application at the left isthmus, local electrogram (Left panel) is markedly attenuated (Middle panel) until notched, split potential and conduction block is recorded (Right panel) before termination of tachycardia. AF, atrial fibrillation; AFI, atrial flutter; CFE, complex fractionated electrograms; CFEA, continuous fractionated electrogram-guided ablation; CS, coronary sinus; PVI, pulmonary vein isolation; RF, radiofrequency.

\section{Mechanisms of Atrial Tachycardias and Results of Catheter Ablation}

A total of 114 atrial tachycardias or flutters were observed during combined PVI and CFEA procedures (60 in PAF, 54 in PeAF patients). The tachycardia mechanisms were focal $(20 / 114,17.5 \%)$, roof-dependent $(20 / 114,17.5 \%)$, peri-mitral $(33 / 114,28.9 \%)$, cavotricuspid isthmus-dependent $(34 / 114$, $29.8 \%)$, or unknown $(7 / 114,6.1 \%)$. The major mechanism of tachycardia was macroreentry around large anatomic obstacles such as the PV and mitral or tricuspid annuli (87/114, $76.3 \%)$. Focal mechanism was responsible in $18.3 \%(11 / 60)$ of tachycardias in PAF patients and in $16.7 \%$ (9/54) of tachycardias in PeAF patients. The mechanism of some tachycardias was difficult to identify because of the coexistence of multiple tachycardias, inconsistent entrainment results, and frequent termination or transition to other tachycardia during entrainment pacing. Table 3 summarizes the tachycardia mechanisms and results of catheter ablation. Successful ablation was achieved in 93/114 cases (81.6\%) (Table 3).

\section{Clinical Results and Complications}

Complications comprised transient ischemic attack in 1, pericardial effusion requiring drainage in 2 , and groin hematoma in 2 patients.

The 70 PAF patients had a mean follow-up of $17 \pm 10$ months. Recurrent tachyarrhythmias occurred in 11 patients, comprising persistent AFl (3 patients), PAF (7 patients), and AV nodal reentry tachycardia (AVNRT: 1 patient). Repeat procedures were performed in $1 \mathrm{AVNRT}, 3 \mathrm{AFl}$ and in $4 \mathrm{PAF}$ patients. Atrial arrhythmias did not recur after this second procedure in $6 / 7$ patients. One patient experienced recurrent episodes of PAF after the second procedure and 3 patients refused to undergo a redo ablation: 3 of these 4 patients were managed with intermittent ( 1 patient) or regular ( 2 patients) medication with class IC antiarrhythmic agents. In 1 patient, AF became persistent, and a rate control strategy was used. 
The 35 PeAF patients had a mean follow-up of $20 \pm 9$ months: 18 patients developed persistent $\mathrm{AFl}$ (5 patients) or PeAF (13 patients) and of those, $5 \mathrm{AFl}$ patients and $7 \mathrm{AF}$ patients underwent repeat procedures. The remaining $6 \mathrm{AF}$ patients refused to undergo a second procedure. All the $5 \mathrm{AFl}$ patients and 2 of the 7 peAF patients regained sinus rhythm after the second procedure. In $2 / 7$ patients who underwent redo procedures, and in $1 / 6$ patients who did not receive redo procedure, previously ineffective class IC antiarrhythmic agents were effective for maintaining sinus rhythm. For 3 patients in whom AF recurred after the second procedure and 5 of the 6 patients who refused a repeat procedure, a rate control strategy was used. Overall, sinus rhythm was maintained without antiarrhythmic agents in $24(69 \%)$ patients, and with previously ineffective antiarrhythmic drugs in $3(9 \%)$ patients. $\mathrm{AF}$ persisted in the remaining $8(23 \%)$ patients.

\section{Discussion}

The present study investigated the incidence and mechanism of regular ATA occurring during the combined approach of PVI and CFEA in catheter ablation of AF. We found that CFEA after PVI was able to render AF non-inducible in PAF patients or to convert AF to AFl in PeAF patients. The major mechanism underlying induced or converted AFls during this combined procedure was macroreentry around large anatomic obstacles such as the tricuspid/mitral annulus or PVs. This result suggests that it is possible to take a step-wise, patientspecific approach based on individual differences in the electrophysiological substrate of AF.

\section{Efficacy of Post-PVI CFEA and Its Implication on the Mechanism of AF}

The inducibility of AF by pacing is a major determinant of recurrence after $\mathrm{PV}$ isolation. ${ }^{11-14}$ The recurrence rate ranges from $30 \%$ to $50 \%$ when $\mathrm{AF}$ remains inducible, as compared to $10-20 \%$ when non-inducibility is achieved. In the present study, AF became non-inducible with PVI alone in $55.7 \%$ of PAF patients, and became non-inducible in a further $31.4 \%$ of patients after additional CFEA. In PeAF patients, PVI alone was not successful in terminating AF in any patient, wheras additional CFEA resulted in AF termination into sinus rhythm in $5.7 \%$ of patients or into $\mathrm{AFl}$ in $60.0 \%$ of patients. Therefore, while the long-term success of these CFEA outcomes remains unknown, CFEA resulted in greater achievement of the procedural endpoint.

The results of the CFEA provide 2 important mechanistic implications for the maintenance of AF. First, the fact that CFEA renders inducible AF non-inducible or into inducible AFl implies that the region targeted for CFEA could have played an active role in the perpetuation of AF. Second, the transition or termination of PeAF into $\mathrm{AFl}$ and subsequent revelation of macro-reentrant or focal tachycardia suggests that AF comprises different subsets of arrhythmia mechanisms. The focal or micro-reentrant mechanisms around or within the PV antrum are eliminated by a debulking PVI procedure. Further reduction of the arrhythmia burden is achieved by CFEA, leaving a few macroreentrant or focal tachycardias at the final stage of the ablation procedure. The stepwise transition of atrial arrhythmias during this combined procedure suggests a stepwise reduction of the arrhythmia burden that maintains AFl.

Anatomical Distribution of Fractionated Electrograms in AF Fractionated electrograms targeted for ablation were dis- tributed most frequently in the LA roof, septum, mitral annulus and appendage. Anatomical landmarks harboring these abnormal electrograms were similar to those proposed by Nademanee et $\mathrm{al}^{7}$ and Haissaguerre et al. ${ }^{15}$ The distribution of these abnormal electrograms were similar in patients with paroxysmal or PeAF. These anatomical structures have been shown to consist of heavily intermingled atrial fibers of septopulmonary and septoatrial bundles with abrupt changes and overlap in the fibers orientation. ${ }^{16}$ This structural distinction provides the anatomical basis for formation of reentrant arrhythmia. However, the possibility of bystander complex electrograms that do not play an active role but are generated passively by the inherently abnormal fiber connections, and thus generating a pseudo-CFE, should also be considered.

\section{Mechanism of Regular Tachycardia Induced or Converted From AF During the Combined Ablation Procedure}

Inducible $\mathrm{AF}$ was converted into inducible $\mathrm{AFl}$ in 21 of the $70 \mathrm{PAF}$ patients who underwent PVI and/or CFEA, while AF terminated into $\mathrm{AFl}$ in 21 of the $35 \mathrm{PeAF}$ patients. The majority $(87 / 114,76.3 \%)$ of the tachycardias were peri-mitral, roof-dependent peri-PV or cavotricuspid isthmus-dependent tachycardias, which emphasizes the importance of large anatomical barriers. Focal tachycardia frequently originated from the posterior wall or the septum. Successful ablation was possible in $95 \%$ of the focal tachycardias.

Differential diagnosis of these regular tachycardias was sometimes difficult because of the coexistence of multiple tachycardias, frequent and subtle changes in the activation sequence or tachycardia CLs during entrainment pacing. ${ }^{17}$ Focal tachycardias, because of previous ablation lines (roof line, mitral or cavotricuspid isthmus line), frequently showed CL coverage greater than $50 \%$ and mimicked reentrant tachycardia. An integrative analysis of activation and entrainment mapping results was necessary for differential diagnosis of these tachycardias.

\section{Study Limitations}

We did not systematically map all areas of fractionated electrograms before ablation, which might have precluded description of the global distribution of fractionated electrograms or their changes after ablation. A 3D mapping system-guided identification of CFE is currently underway in our laboratory to display the location and distribution of CFE areas.

The data describe the immediate results of CFEA. To verify the true nature of this incremental change, a randomized study comparing the various strategies is required (ie, PVI alone vs CFEA alone vs PVI+CFEA).

Although the additive effect of CFEA is described, it remains unclear whether this resulted from abolition of AF drivers or from non-specific atrial damage and atrial mass reduction. In addition, not all of the areas with fractionated electrograms may represent true AF drivers. Future studies should obtain more data regarding the nature of electrograms at or near the AF drivers in order to reduce unnecessary RF application.

Finally, the clinical outcome was judged by documented ECGs or Holter recordings obtained intermittently during outpatient clinic follow-up or emergency room visits. A more comprehensive follow-up program, such as trans-telephonic transmission or event recording, could have detected more cases of asymptomatic occurrence of AF in our study subjects and resulted in a lower success rate. 


\section{Conclusion}

CFEA after PVI resulted in non-inducibility of AF or conversion of inducible AF into inducible AFl in patients with PAF. CFEA terminated AF into sinus rhythm or into AFl in patients with PeAF. The major mechanism of the induced or converted AFls during this combined procedure was macroreentry around large anatomical obstacles such as the tricuspid/mitral annulus or PVs.

\section{Acknowledgments}

This work was supported in part by grants from the Asan Institute for Life Science (2006-232) and the Korean Institute of Medicine.

We thank Jin-Yong Jung and Sung-Ho Moon for their technical assistance, and Jeong-Hae Kwon, RN, Ji-Hoi Yoon, RN and Ji-Hyun Kim, RN for their support with the preparation of this manuscript.

\section{References}

1. Haïssaguerre M, Jaïs P, Shah DC, Takahashi A, Hocini M, Quiniou $\mathrm{G}$, et al. Spontaneous initiation of atrial fibrillation by ectopic beats originating in the pulmonary veins. N Engl J Med 1998; 339: 659666.

2. Haïssaguerre $M$, Shah DC, Jaïs $P$, Hocini $M$, Yamane $T$, Deisenhofer I, et al. Electrophysiological breakthroughs from the left atrium to the pulmonary veins. Circulation 2000; 102: $2463-$ 2465 .

3. Oral H, Pappone C, Chugh A, Good E, Bogun F, Pelosi F Jr, et al. Circumferential pulmonary-vein ablation for chronic atrial fibrillation. N Engl J Med 2006; 354: 934-941.

4. Jalife J, Berenfeld O, Mansour M. Mother rotors and fibrillatory conduction: A mechanism of atrial fibrillation. Cardiovasc Res 2002; 54: 204-216.

5. Sanders P, Berenfeld O, Hocini M, Jaïs P, Vaidyanathan R, Hsu LF, et al. Spectral analysis identifies sites of high-frequency activity maintaining atrial fibrillation in humans. Circulation 2005; 112: $789-797$.

6. Kumagai K, Nakashima H. Noncontact mapping-guided catheter ablation of atrial fibrillation. Circ J 2009; 73: 233-241.

7. Nademanee K, McKenzie J, Kosar E, Schwab M, Sunsaneewitayakul B, Vasavakul T, et al. A new approach for catheter ablation of atrial fibrillation: Mapping of the electrophysiologic substrate. J Am Coll Cardiol 2004; 43: 2044-2053.

8. Oral H, Chugh A, Good E, Sankaran S, Reich SS, Igic P, et al. A tailored approach to catheter ablation of paroxysmal atrial fibrillation. Circulation 2006; 113: 1824-1831.

9. Konings KT, Smeets JL, Penn OC, Wellens HJ, Allessie MA. Configuration of unipolar atrial electrograms during electrically induced atrial fibrillation in humans. Circulation 1997; 95: 1231-1241.

10. European Heart Rhythm Association (EHRA); European Cardiac Arrhythmia Scoiety (ECAS); American College of Cardiology (ACC); American Heart Association (AHA); Society of Thoracic Surgeons (STS), Calkins H, Brugada J, Packer DL, Cappato R, Chen SA, Crijns HJ, et al. HRS/EHRA/ECAS expert Consensus Statement on catheter and surgical ablation of atrial fibrillation: Recommendations for personnel, policy, procedures and followup. A report of the Heart Rhythm Society (HRS) Task Force on catheter and surgical ablation of atrial fibrillation. Heart Rhythm 2007; 4: 816-861.

11. Jaï $P$, Hocini M, Sanders P, Hsu LF, Takahashi Y, Rotter M, et al. Long-term evaluation of atrial fibrillation ablation guided by noninducibility. Heart Rhythm 2006; 3: 140-145.

12. Oral H, Chugh A, Lemola K, Cheung P, Hall B, Good E, et al. Noninducibility of atrial fibrillation as an end point of left atrial circumferential ablation for paroxysmal atrial fibrillation: A randomized study. Circulation 2004; 110: 2797-2801.

13. Oral H, Knight BP, Ozaydin M, Tada H, Chugh A, Hassan S, et al. Clinical significance of early recurrences of atrial fibrillation after pulmonary vein isolation. J Am Coll Cardiol 2002; 40: 100-104.

14. Haïssaguerre M, Sanders P, Hocini M, Hsu LF, Shah DC, Scavée $\mathrm{C}$, et al. Changes in atrial fibrillation $\mathrm{CL}$ and inducibility during catheter ablation and their relation to outcome. Circulation 2004; 109: $3007-3013$.

15. Haïssaguerre M, Sanders $\mathrm{P}$, Hocini $\mathrm{M}$, Takahashi $\mathrm{Y}$, Rotter $\mathrm{M}$, Sacher F, et al. Catheter ablation of long-lasting persistent atrial fibrillation: Critical structures for termination. J Cardiovasc Electrophysiol 2005; 16: 1125-1137.

16. Ho SY, Anderson RH, Sánchez-Quintana D. Atrial structure and fibres: Morphologic bases of atrial conduction. Cardiovasc Res 2002; 54: 325-336

17. Ohkubo K, Watanabe I, Yamada T, Okumura Y, Hashimoto K, Ashino S, et al. P wave morphology of an arrhythmogenic focus in patients with atrial fibrillation originating from a pulmonary vein or the superior vena cava. Circ J 2008; 72: 1650-1657. 